\title{
Les pratiques évaluatives d'enseignants en soins infirmiers lors des stages : une étude descriptive qualitative
}

\author{
Teachers' assessment practices in context of nursing clinical education : \\ a qualitative descriptive study
}

\section{Patricia BLANCHETTE}

MD en sciences infirmières, Cegep de Chicoutimi, Chicoutimi, Québec, Canada

\section{Maud-Christine CHOUINARD}

Professeur titulaire, Université du Québec, Chicoutimi

\section{Jacques-André GUEYAUD}

Professeur titulaire chercheur, Université du Québec, Chicoutimi

\begin{abstract}
RÉSUMÉ
Aucune étude recensée n'a permis de dresser le portrait québécois des pratiques évaluatives d'enseignants en soins infirmiers dans le contexte des stages en formation collégiale. Compte tenu de l'importance du stage et de l'évaluation dans la formation de l'étudiant en soins infirmiers, cette étude s'avère justifiée. Cet article présente les résultats d'une étude visant à décrire et comprendre les pratiques évaluatives d'enseignants lors d'évaluations en cours d'apprentissage d'étudiants en stage et leur contexte de réalisation, ainsi qu'à identifier les facteurs influençant ces pratiques. Dans le cadre d'une recherche qualitative descriptive, la collecte des données a reposé principalement sur la description de pratiques rapportées et recueillies lors d'entrevues réalisées auprès de 10 enseignantes en soins infirmiers d'un cégep de la région du Saguenay-Lac-Saint-Jean (SLSJ, Québec, Canada) soit : l'observation directe et indirecte; les questions posées à l'étudiant et au groupe d'étudiants et la rétroaction individuelle et de groupe. Ils ont aussi permis d'énumérer et de catégoriser les facteurs pouvant influencer ces pratiques, soit les facteurs facilitants, les barrières et les croyances.
\end{abstract}

Mots clés : évaluation des acquis scolaires, enseignement infirmier, recherche qualitative, élève infirmier, internat spécialité paramédicale.

Pour citer I'article:

Blanchette P, Chouinard MC, Gueyaud JA. Les pratiques évaluatives d'enseignants en soins infirmiers lors des stages : une étude descriptive qualitative. Rech Soins Infirm. 2017 Jun;(129):60-72. 
Since no study has yet been able to assess the Quebec portrait of teachers' evaluation practices in the context of clinical education in nursing. Considering the importance of clinical teaching and evaluation in the education of nursing students, this study is justified. This article presents the results of a study that aimed to describe and understand the experience of teachers in the ongoing assessment of nursing students' learning during clinical education. It aimed to describe the evaluation practices of teachers during the learning of students in clinical education and achievement context, and to identify factors influencing these practices. In the context of descriptive qualitative research, the data collection was mainly based on the description of practices reported and collected during interviews realized with 10 nursing teachers of a college of the region of Saguenay-Lac-Saint-Jean (SLSJ, Quebec, Canada). The results allowed, for instance, to identify and to describe the evaluation practices led in this specific context of the study : the direct and indirect observation ; the questions asked to the sudent and to the group of sutdents and individual and group feed-back. They also allowed to enumerate and to categorize factors which can influence these practices, that is facilitates factors, barriers and faiths.

Key words : measurement, education Nursing, qualitative research, students nursing, internship nonmedical.

\section{Remerciements}

Les auteurs tiennent à remercier toutes les personnes ayant contribué de près ou de loin à cette étude. Une attention particulière est portée à l'intention des participants à l'étude qui se sont montrés disponibles, qui ont partagé leur expérience, et sans qui cette étude n'aurait pu être achevée.

\section{INTRODUCTION}

Au Québec, la formation infirmière est offerte à deux niveaux d'enseignement distincts, soit collégial dans différents collèges d'enseignement général et professionnel ou cégeps (enseignement collégial) soit universitaire, dans diverses universités (enseignement universitaire). La formation collégiale comprend deux types de formations : la formation régulière d'une durée de trois ans et la formation intensive d'une durée approximative de 18 mois. Ces formations respectent le plancadre de formation émis par le Ministère de l'éducation et de l'enseignement supérieur (MÉES) du Québec, soit le programme Soins infirmiers 180-A0. II comprend un maximum de 1035 heures de stages dont la répartition dans le programme varie d'un cégep à l'autre. Le plus souvent, le groupe d'étudiants en stage est sous la supervision directe d'un enseignant du cégep. La supervision indirecte soit le jumelage d'une infirmière avec un étudiant dans le milieu de stages, est un mode de supervision également employé par les cégeps québécois. La charge d'enseignement et les milieux cliniques choisis sont variables d'un cégep à l'autre. Cependant, la médecine, la chirurgie, la pédiatrie, l'obstétrique et la psychiatrie sont des passages obligés.
Les pratiques évaluatives des enseignants en soins infirmiers mises en œuvre dans l'évaluation des apprentissages en stage n'ont pas été étudiées dans le cadre de l'enseignement collégial au Québec. Bien que certaines études et écrits se soient penchés sur l'évaluation des apprentissages au collégial au Québec, peu concernaient l'évaluation des apprentissages en stage (1). Parmi ces études, aucune ne traite des pratiques évaluatives d'enseignants en soins infirmiers dans le contexte collégial au Québec.

Les stages occupent une place importante dans la formation infirmière (2) et ils contribuent au développement des compétences nécessaires à l'exercice de la profession (3). L'enseignant qui accompagne un étudiant en stage, a des missions d'enseignement et d'évaluation $(4,5)$ de l'atteinte des compétences visées par le stage. La manière dont l'évaluation en cours d'apprentissage est mise en œuvre par l'enseignant peut offrir des régulations riches à l'étudiant et, de cette façon, contribuer au développement de ses compétences et à sa réussite. Pour la présente étude, un cadre de référence a été conceptualisé par les auteurs à partir de références faisant partie de la recension des écrits réalisée. Ce cadre situe les différents concepts entourant 
l'évaluation diagnostique et l'évaluation formative examinés en lien avec la régulation souhaitée lors de l'évaluation en cours de stage.

L'importance de l'évaluation en cours d'apprentissage pour le développement des compétences de l'étudiant et l'absence de données relatives aux pratiques justifient la conduite d'une étude descriptive qualitative. Cette étude a comme but de décrire et comprendre les pratiques évaluatives d'enseignants lors de l'évaluation en cours d'apprentissage auprès des étudiants en stage et leur contexte de réalisation ainsi qu'identifier les facteurs d'influence de ces pratiques. Une meilleure compréhension de ces pratiques constitue une étape essentielle à leur amélioration. Cette étude vise deux objectifs :

1) Dresser le panorama des pratiques évaluatives en cours de stage à partir du discours des enseignants en soins infirmiers d'un cégep de la région du Saguenay-Lac-SaintJean (SLSJ) ;

2) Identifier les facteurs influençant les pratiques ainsi déclarées par les enseignants en soins infirmiers d'un cégep de la région du SLSJ ?

\section{MATÉRIEL ET MÉTHODES}

\section{Devis}

Pour répondre à ces objectifs, une approche descriptive qualitative (6) a été adoptée. Sandelowski énonce que la recherche descriptive qualitative est la méthode idéale pour décrire les expériences personnelles et les réponses des personnes à un événement ou une situation (7). De plus, un devis qualitatif s'avère tout à fait indiqué pour explorer des phénomènes peu étudiés, comme c'est le cas dans cette présente étude. Le paradigme interprétatif/descriptif a donc guidé les choix méthodologiques de cette étude descriptive qualitative puisqu'il explore la manière dont chaque personne donne du sens de façon subjective à sa réalité et les significations qu'elle y attribue (8).

\section{- Milieu de l'étude}

Bien que le phénomène étudié soit vécu dans d'autres cégeps du Québec, l'étude s'est limitée à un cégep de la région du SLSJ, au Québec, Canada. Étant donné l'absence de données sur le phénomène à l'étude, le choix du milieu a visé l'obtention d'un premier panorama dans un milieu précis.

Le milieu à l'étude est un cégep public du SLSJ employant 600 personnes avec une inscription annuelle d'environ 3000 étudiants. Il offre cinq programmes d'études préuniversitaires et 17 programmes d'études techniques. À l'automne 2015, 28 enseignants (formation régulière) et 12 chargés de cours (formation intensive) en soins infirmiers étaient employés dans ce cégep. Le mode d'encadrement employé pour les stages en soins infirmiers est la supervision directe. Pour une session donnée, un enseignant supervise un groupe constitué de six étudiants au maximum sur en moyenne 36 jours de stages.

\section{Population et échantillon}

Les 40 enseignants et chargés de cours en soins infirmiers du cégep à l'étude ont été approchés pour participer à l'étude au mois d'avril 2016. Un échantillonnage non probabiliste de type accidentel (6) a permis de constituer un échantillon de 10 participants. Les enseignants recrutés devaient répondre aux critères d'inclusion suivants : 1) être enseignant ou chargé de cours en soins infirmiers (formation régulière ou intensive) ; et 2) avoir supervisé au moins un groupe d'étudiants en stage de soins infirmiers. Afin de favoriser la participation, aucun critère de non inclusion n'a été retenu.

\section{Approbation éthique}

Des approbations pour le déroulement de cette étude ont été obtenues de la direction générale du cégep à l'étude ainsi que du Comité d'éthique de la recherche de l'Université du Québec à Chicoutimi (UQAC). Au début de chaque entrevue, les deux copies du formulaire d'information et de consentement ont été signées par le participant et le chercheur principal.

\section{Déroulement de la collecte des données}

Les participants potentiels ont été informés de l'étude au cours d'une assemblée départementale et sollicités par courriel. La première étape de collecte des données a eu lieu lors d'entrevues initiales individuelles semi-structurées. Ces entrevues, d'une durée moyenne de 60 minutes, se sont déroulées dans un endroit calme, neutre et en face à face. Ensuite, un questionnaire contenant des questions d'ordre sociodémographique a été complété par le participant afin d'obtenir des données descriptives de l'échantillon. Pour l'entrevue initiale, un guide d'entrevue a été élaboré et approuvé au préalable par un comité d'experts en enseignement des soins infirmiers $(n=2)$ et par les directeurs de recherche. Les questions ouvertes contenues dans le guide ont permis de mener à bien les entrevues tout en laissant assez de souplesse à l'interviewer pour explorer davantage certains aspects. Les entrevues ont été enregistrées de façon audionumérique puis retranscrites en entier en verbatim par l'interviewer. Un journal de bord a été complété afin de saisir et de retenir des données vues et ressenties pendant l'entrevue et pour guider les entrevues subséquentes (9), mais également pour maintenir la distanciation nécessaire à la réduction de la subjectivité de l'interviewer (10).

Enfin, la dernière étape de collecte de données a eu lieu lors d'entrevues de validation. Pour ce faire, les résultats sommaires ont été remis aux participants de façon individuelle, puis ils ont été rencontrés en face à face lors 


\section{Les pratiques évaluatives d'enseignants en soins infirmiers lors des stages : une étude descriptive qualitative}

d'entrevues semi-dirigées d'une durée approximative de 30 minutes. Ces entrevues de validation ont permis de recueillir les impressions des participants à l'égard des résultats, de la participation au projet et aussi, d'ajouter ou de préciser certains propos. Afin de s'assurer de ne perdre aucune donnée, les entrevues de validation ont été enregistrées de façon audionumérique, mais non pas été retranscrites. Les données amassées de la sorte ont été consignées dans le journal de bord de l'interviewer suite à l'écoute des entrevues puis ont été ajoutées aux analyses.

\section{Analyse des données}

Dans le but de s'imprégner des données amassées, la collecte des données et la retranscription des entrevues se sont faites dans la même période. Les notes du journal de bord ont été ajoutées à l'analyse sous forme d'annotations à la retranscription correspondante. La codification et l'analyse des données ont été réalisées à l'aide du logiciel NVivo version 11. L'analyse des données a été effectuée selon les trois étapes suggérées par Miles et al. (11). : 1) condensation des données, 2) présentation des données, et 3) élaboration et vérification des données. La condensation des données a débuté lors de la retranscription des entrevues initiales. Ensuite, la lecture des transcriptions a permis de dégager les extraits et les thèmes qui semblaient pertinents (codification). Par la suite, un arbre thématique a été élaboré de façon progressive. Le choix des thèmes généraux et les sousthèmes qui le composent a été guidé par les transcriptions (approche inductive), ainsi que par le cadre de référence et le guide d'entrevue (approche déductive). La présentation des données s'est faite sous forme de tableaux qui ont évolué tout au long du processus d'analyse et ont été modifiés en fonction des discussions de l'équipe de recherche. Ainsi, l'élaboration et la vérification des données se sont effectuées tout au long du processus d'analyse par différentes méthodes, entre autres par des discussions entre chercheurs.

\section{Critères de rigueur scientifique}

L'appréciation de la qualité de cette étude en regard des critères de rigueur scientifique spécifiques à la recherche qualitative ont été inspirés des travaux de Laperrière (9). La crédibilité de l'étude a été assurée entre autres par les éléments suivants : l'engagement d'un des chercheurs dans le milieu, les notes du journal de bord, une codification exhaustive, la validation des résultats auprès des participants et la prise en compte de la subjectivité (distanciation, réflexivité, discussion entre chercheurs). L'engagement prolongé du chercheur est la meilleure façon d'assurer la crédibilité d'une étude $(8,12)$. Pour certains chercheurs, la transférabilité des données est envisageable en recherche qualitative $(9,10)$. Dans ce contexte, la description exhaustive du milieu à l'étude et des caractéristiques des participants ainsi que l'exploration en profondeur du phénomène permettent d'assurer le critère de transférabilité pour des milieux similaires $(9,10)$. Pour répondre au critère de fiabilité et de confirmabilité, les entrevues ont été analysées en équipe. Par ailleurs, les résultats des analyses préliminaires ont été validés.

\section{RÉSULTATS}

Pour permettre de comparer les résultats obtenus, les caractéristiques jugées importantes des participants sont d'abord présentées. Ensuite, la description des pratiques évaluatives d'enseignants lors de l'évaluation en cours d'apprentissage d'étudiants en contexte de stage de soins infirmiers est proposée. Finalement, comme les résultats n'en permettent pas une description exhaustive, les facteurs d'influence soit les barrières, les facteurs facilitants et les croyances de ces pratiques sont énumérés et catégorisés.

\section{Caractéristiques générales des participants}

Tous les participants étant des femmes, le féminin sera employé pour les désigner. La majorité d'entre elles ont un poste permanent d'enseignante en soins infirmiers (7/10) et leur nombre d'années d'expérience à ce titre est assez varié. La majorité a supervisé plus de 23 jours de stages par session dans les trois années de formation (7/10). Huit participantes ont complété leur baccalauréat en sciences infirmières et de la santé (premier cycle universitaire), alors que les deux autres l'ont débuté. De plus, elles ont toutes suivi des formations en pédagogie, mais de nature et durée différentes. Seulement deux des participantes ont réalisé des études dans d'autres domaines que les soins infirmiers et l'enseignement. Avant d'enseigner, les enseignantes interrogées ont exercé la profession d'infirmière dans au moins trois services distincts en milieu hospitalier. La majorité (7/10) supervise des stages dans cinq milieux cliniques différents ou plus, le maximum étant de dix milieux $(1 / 10)$ et le minimum de trois $(2 / 10)$.

\section{Description des pratiques évaluatives}

Les données colligées ont permis de décrire les pratiques évaluatives mises en œuvre par les enseignantes interrogées lors de l'évaluation en cours d'apprentissage d'étudiants en contexte de stage de soins infirmiers. Pour un stage donné, les pratiques étudiées sont celles comprises dans la période débutant après le début du stage et terminant avant la fin du stage. Elles ne comprennent pas celles réalisées avant le stage (définition des objectifs de stage) et à la fin du stage (évaluation certificative). Ces résultats sont énumérés dans le tableau 1. Ces pratiques évaluatives ont été organisées à partir d'une catégorisation qui a émergé au cours de l'analyse des données, puis qui a pu être appuyée à partir de différents auteurs (1, 13-19). Dans cette catégorisation cadre, les pratiques évaluatives ont été divisées en deux catégories, soit la « collecte d'information » et l'« information transmise à l'étudiant » puisqu'il s'agit selon Roland (13) des deux volets du processus de l'évaluation des apprentissages dans la 


\begin{tabular}{|c|c|c|c|c|}
\hline \multicolumn{5}{|c|}{ Pratiques évaluatives lors de l'évaluation en cours d'apprentissage } \\
\hline \multirow{2}{*}{\multicolumn{3}{|c|}{ Collecte d'information }} & \multirow{2}{*}{\multicolumn{2}{|c|}{$\begin{array}{c}\text { Information transmise à l'étudiant } \\
\text { Rétroactions }\end{array}$}} \\
\hline & & & & \\
\hline $\begin{array}{l}\text { Observation } \\
\text { directe }\end{array}$ & Observation indirecte & Questions & Individuelles & De groupe \\
\hline $\begin{array}{l}\text { - Observations } \\
\text { de faits et de } \\
\text { représentations ; } \\
\text { - Observations } \\
\text { de l'étudiant en } \\
\text { situations de } \\
\text { stage. }\end{array}$ & $\begin{array}{l}\text { - Observations rapportées par } \\
\text { une autre enseignante ; } \\
\text { - Observations rapportées par un } \\
\text { autre étudiant ; } \\
\text { - Observations rapportées par } \\
\text { un client ou un membre de sa } \\
\text { famille ; } \\
\text { - Observations rapportées par } \\
\text { un professionnel du milieu } \\
\text { clinique ; } \\
\text { - Observations consignées dans } \\
\text { le contrat d'apprentissage de } \\
\text { l'étudiant ; } \\
\text { - Observations faites lors de la } \\
\text { révision des notes au dossier et } \\
\text { autres documents du client par } \\
\text { l'étudiant ; } \\
\text { - Observations faites lors de la } \\
\text { vérification de la feuille de route } \\
\text { de l'étudiant ; } \\
\text { - Observations faites dans les } \\
\text { travaux de recherche. }\end{array}$ & $\begin{array}{l}\text { - Questions } \\
\text { posées à } \\
\text { l'étudiant ; } \\
\text { - Questions } \\
\text { posées } \\
\text { au groupe } \\
\text { d'étudiants. }\end{array}$ & $\begin{array}{l}\text { - Rétroactions individuelles } \\
\text { planifiées à mi-stage (orales } \\
\text { et/ou écrites); } \\
\text { - Rétroactions individuelles } \\
\text { planifiées à la demande de } \\
\text { l'étudiant (orales ou écrites); } \\
\text { - Rétroactions individuelles } \\
\text { planifiées lors d'entretien(s) } \\
\text { de confrontation (orales et } \\
\text { écrites); } \\
\text { - Rétroactions individuelles } \\
\text { ponctuelles (orales); } \\
\text { - Rétroactions individuelles } \\
\text { ponctuelles par les pairs } \\
\text { (orales); } \\
\text { - Rétroactions ponctuelles } \\
\text { ou planifiées effectuées } \\
\text { par l'étudiant lui-même } \\
\text { (autorégulation) ; } \\
\text { - Rétroactions individuelles } \\
\text { écrites dans les travaux de } \\
\text { stage ; } \\
\text { - Rétroactions individuelles } \\
\text { écrites sur la grille } \\
\text { d'évaluation. }\end{array}$ & $\begin{array}{l}\text { - Rétroactions } \\
\text { ponctuelles } \\
\text { de groupe ; } \\
\text { - Rétroactions } \\
\text { de groupe } \\
\text { planifiées } \\
\text { lors d'un } \\
\text { débriefing ; } \\
\text { - Rétroactions } \\
\text { de groupe } \\
\text { planifiées lors } \\
\text { de rencontres } \\
\text { de groupe. }\end{array}$ \\
\hline
\end{tabular}

Tableau 1:

Pratiques rapportées en lien avec l'évaluation pendant le stage

pratique courante des enseignants. La collecte d'information comprend l'observation (1, 14-16), qui peut être directe ou indirecte (1) et les questions adressées à l'étudiant (15). Les différents types de rétroactions, soit individuelle ou de groupe, réfèrent à l'information transmise à l'étudiant.

Les pratiques de collecte d'informations rapportées relèvent de l'observation directe (en présence), l'observation indirecte (informations provenant d'autres sources) et les questions adressées à l'étudiant ou au groupe d'étudiants. La façon qu'a l'enseignant d'informer l'étudiant de sa réaction face à sa réponse ou la démarche utilisée lors d'une situation réelle vécue durant le stage correspond à la rétroaction. Elle lui est adressée de façon individuelle ou en groupe et sont regroupées de la façon suivante : les pratiques de rétroaction individuelle, à savoir, les rétroactions individuelles planifiées à mi-stage (orales ou écrites ou les deux) ; les rétroactions individuelles planifiées faites à la demande de l'étudiant (orales ou écrites) ; les rétroactions individuelles planifiées lors d'entretiens de confrontation (orales et écrites) ; les rétroactions individuelles ponctuelles (orales); les rétroactions individuelles ponctuelles par les pairs (orales); les rétroactions ponctuelles ou planifiées effectuées par l'étudiant lui-même (rétroaction interne); les rétroactions individuelles écrites dans les travaux de stage et les rétroactions individuelles écrites sur la grille d'évaluation et les pratiques de rétroaction de groupe à savoir les rétroactions ponctuelles de groupe, les rétroactions de groupe lors de débriefing et les rétroactions de groupe lors de rencontre post-stage (après une journée de stage).

Les outils d'évaluation étant considérés comme des dispositifs permettant de recueillir les informations nécessaires au jugement sur la performance de l'étudiant et/ou qui permettent 


\section{Les pratiques évaluatives d'enseignants en soins infirmiers lors des stages : une étude descriptive qualitative}

de documenter la performance de l'étudiant (17), ceux utilisés pendant le stage par les enseignantes interrogées ont également été répertoriés.

Pratiques de collecte d'information. La première catégorie des pratiques de collecte d'information relative à l'évaluation en cours d'apprentissage des étudiants est l'observation directe. L'observation directe correspond au moment où l'enseignante observe directement un étudiant en train d'agir dans une situation naturelle (milieu de stage réel) en situation non manipulée (non créée). L'observation en présence constitue la manière d'obtenir de l'information sur l'étudiant lui-même. L'observation directe de l'étudiant comprend également son environnement et les personnes avec qui il interagit. Pour ce faire, l'enseignante peut se trouver à proximité de l'étudiant ou à distance (cadre de porte, de l'autre côté du rideau, au chevet d'un autre client, au poste des infirmières). L'observation directe peut se faire en plusieurs lieux, en fonction de l'endroit où se trouve l'étudiant dans le département (chambre du client, poste des infirmières, corridor) et ce tout au long de la journée de stage et de la période de stage complète. Un étudiant fait l'objet d'observation directe à une fréquence très variable. Certaines enseignantes observent plus ou moins souvent en fonction du niveau de l'étudiant. "Si c'est la première fois qu'elle fait la technique et que moi c'est la première fois que je la vois aller dans une technique [en parlant de l'étudiante] c'est certain que je vais y aller [en parlant de l'observation] [...] Première année, je vais superviser tout [...] mais en sixième session, non je n'irai pas nécessairement voir la première fois qu'elle va faire une prise de sang [...] tout dépendant d'où ils sont rendus [en parlant du niveau de l'étudiant] » (Part-07). Aussi, certaines rapportent qu'elles procèdent à l'observation de façon systématique lorsque l'étudiant entreprend des actions spécifiques qu'elles jugent à risques pour le client, à titre d'exemple : l'administration de la médication, la réfection de pansements complexes, l'administration de l'hyperalimention intraveineuse, les soins de stomies. Cette pratique augmente en fréquence pour certaines lorsque l'étudiant est en difficulté. En général l'observation directe est mise en œuvre par les enseignantes interrogées afin de permettre d'amasser les informations nécessaires à l'évaluation, de faire une régulation à l'étudiant ou l'enseignant, d'assurer la sécurité du client ou parce que l'étudiant lui communique le besoin d'être observé.

Une participante a décrit l'observation directe de l'étudiant de cette manière : “ Si c'est la première fois qu'elle [en parlant d'une étudiante] fait la technique et que moi c'est la première fois que je la vois aller dans une technique, c'est sûr que je vais y aller. Souvent, les premières journées, on va évaluer, voir comment c'est et à quel niveau ils [en parlant des étudiants] sont rendus. En première année, je vais superviser tout. Je vais même superviser le bain et je vais leur demander de venir me chercher pour laver le dos. Mais en sixième session, non je n'irai pas nécessairement voir la première fois qu'elle [en parlant d'une étudiante] va faire une prise de sang. Je vais lui demander : c'est quoi ta technique ? [...] Explique-moi ta technique, comment tu vas faire ça ? C'est beau, parfait, vasy. [...] Aussi je vais évaluer comme une première technique, je vais voir sa façon d'être avec le client, sa façon d'être consciencieuse » (Part-07).

La deuxième catégorie des pratiques de collecte d'information est l'observation indirecte. Elle comprend les sources d'information suivantes : une autre enseignante ; un autre étudiant du groupe ; le client ou un membre de sa famille ; un membre du personnel associé au milieu clinique ; le contrat d'apprentissage (s'il y a lieu) ; le dossier et les autres documents du client ; la feuille de route de l'étudiant et les travaux de recherche de l'étudiant. Une participante décrit ainsi les observations faites lors de la révision des notes au dossier et des autres documents du client par l'étudiant ainsi : «Bien, tu peux objectiver des choses justes en vérifiant son dossier, tu peux objectiver des choses sans être collée sur lui [en parlant de l'étudiant] par ce qu'il te présente [...] » (Part-01). Ces sources d'information comprennent aussi des informations qui ont été observées par d'autres personnes (client-famille, personnel, autre étudiant, autre enseignante) chez l'étudiant et qui sont rapportées à l'enseignante. Ces informations sont obtenues parfois grâce à des observations faites après coup, telles que les travaux de stage, le dossier et les autres documents qui doivent être consignés pour le suivi du client (feuille de route, dossier du client).

Ces observations indirectes se produisent à des fréquences variables, à tout moment de la journée de stage et de la séquence du stage tel qu'en témoigne la participante suivante : «À la fin du stage, et à chaque journée de stage, je fais [...] cela me permet vraiment de prendre mon temps. Je prends 20-30 min avec chaque étudiant à la fin du stage [en parlant de la fin de la journée de stage], puis je réévalue le plan de soins, le dossier, comment il le comprend, comment il a fait sa révision de dossier, sa FADM [fiche d'administration de la médication], ses notes d'observation, comment il a organisé sa journée. Rétrospection, s'il y a des choses que j'ai à revoir [...] » (Part-07). Lorsque plus d'une enseignante supervise un groupe, c'est lors du changement d'enseignante qu'a lieu le partage d'information entre enseignantes. Aussi, il arrive qu'une enseignante prenne des informations à propos d'un étudiant auprès d'une autre enseignante qui l'a supervisé auparavant. Les informations sont recueillies à divers endroits, soit au poste des infirmières, dans la chambre du client, dans la salle de repos des infirmières, au bureau d'une enseignante ou dans un local dédié aux étudiants en milieu clinique. C'est à l'aide de ces différentes sources d'information (faits et représentations), soit des observations indirectes, que les enseignantes rapportent obtenir des informations qui sont nécessaires à l'évaluation. Plus précisément, les motifs rapportés qui justifient l'utilisation de ces différentes sources d'information ont permis d'énumérer les fonctions suivantes : prédictive, formative, régulatrice, descriptive, de vérification et de contrôle de qualité, qui leurs sont attribuées. 
Les questions directement posées à l'étudiant correspondent à une pratique contribuant à la collecte d'information et sont nécessaires à l'évaluation selon les enseignantes entretenues. Les questions sont posées directement à l'étudiant ou au groupe d'étudiants. Pour ce faire, plusieurs méthodes ont été rapportées par ces dernières. L'enseignante peut s'adresser à un étudiant, ou à tout le groupe, et lui demander de répondre instantanément ou le lendemain à une question concernant une situation clinique. Parfois, l'enseignante s'adresse à un étudiant et lui pose des questions sur sa propre perception de sa performance. Également, certaines enseignantes questionnent l'étudiant sur ce qu'il a fait ou ce qu'il va faire. D'autres rapportent qu'elles posent des questions à l'étudiant lors d'une rencontre pour recueillir ses impressions face à son stage en général, sur sa performance, à propos de ses difficultés et pour savoir quelles solutions il envisage pour y remédier. Les enseignantes interrogées ont rapporté poser des questions à des fréquences variables, à différents moments de la journée du stage et de la séquence de stage et dans plusieurs endroits (poste des infirmières, chambre du client, corridor, local fermé et bureau de l'enseignante). Cependant, le moment privilégié par ces dernières pour ce faire, correspond à la révision du (des) dossier(s) de l'étudiant. Les motifs soulevés par les participantes qui justifient l'utilisation de cette pratique permet d'attribuer les fonctions suivantes à cette manière de recueillir l'information : régulation, formative, contrôle de qualité, descriptive et de vérification. Une des participantes justifie cette pratique de la façon suivante : "L'évaluation, tu sais, c'est pas mal plus que les techniques, c'est beaucoup le jugement. Le pourquoi. Donc moi, je questionne toujours le pourquoi. Pourquoi vas-tu faire cela ? Pourquoi as-tu donné cela ? Pourquoi donnes-tu tel médicament ? Quelle évaluation vas-tu faire après ? Dans combien de temps ? » (Part-04).

Pratiques de rétroaction. En ce qui concerne les pratiques reliées à la transmission de l'information à l'étudiant au sujet de l'évaluation, elles comprennent les pratiques de rétroaction individuelle et de rétroaction de groupe. Les sources de rétroaction sont l'enseignante (rétroaction externe), les pairs (rétroaction externe) et l'étudiant lui-même (rétroaction interne). Les rétroactions (information, commentaire ou décision) sont faites de façons verbale, non verbale et écrite. Une rétroaction verbale est décrite par une enseignante de cette façon : « [...] Moi, je dis tout de suite, je n'attends pas ou ce n'est pas vrai que j'attends toujours d'être en dehors de la chambre. Des fois, je vais le dire dans la chambre, puis moi, je considère que c'est du formatif » (Part-01). Alors qu'une rétroaction non verbale est réalisée de cette façon par une autre participante: « Je suis super délicate par rapport à ça. Sauf que mon non verbal à moi il est très, je parle beaucoup sans parler. L'étudiant va le voir juste dans ma face. Normalement, il le sait si ça ne fonctionne pas, je ne parlerai pas devant les étudiants, ni devant les infirmières » (Part-06). Une rétroaction écrite est faite ainsi par une enseignante : «Premièrement, je ne le [en parlant de l'étudiant] corrige vraiment pas de manière exhaustive, j'y vais vraiment avec des petits points, des annotations de un à je ne sais pas combien. Et je demande de préciser, alors je ne corrige pas pour le corriger par-dessus, je mets des points et je pose des questions ? ....]. Cela me permet de voir si l'étudiant est capable d'améliorer. [...] Donc, cela lui permet surtout à lui de faire des liens finalement. Parce qu'il manquait peut-être juste une petite donnée ou un petit quelque chose qu'il n'a pas saisi pour qu'il termine la boucle. .... . Je ramasse le travail, je le corrige, je leur redonne dans la semaine suivante, puis au même moment où je rencontre l'étudiant dans la journée pour lui faire un petit «feed-back » [en parlant de rétroaction], je le regarde avec lui et je lui montre les points qu'il a à développer davantage » (Part-09).

Comme aucune exigence à cet effet n'est demandée par l'établissement et qu'il est laissé à la discrétion de l'équipe d'enseignante de le faire, les rencontres individuelles pour des rétroactions sont parfois planifiées dans une période de temps donnée dans la séquence de stage, par exemple à la moitié du stage (mi-stage). La rétroaction individuelle planifiée à mi-stage est organisée par l'enseignante ou l'étudiant dans un cas et se tient approximativement à mi-stage. Parfois, elle se caractérise par des échanges entre l'étudiant et l'enseignante, alors que d'autres fois, la rétroaction n'est faite qu'à sens unique, c'est-à-dire que ce n'est que l'enseignante qui communique sa perception à l'étudiant. La quasi-totalité des enseignantes pratique la rétroaction individuelle planifiée à mi-stage de façon systématique $(9 / 10)$ alors qu'une autre le fait seulement si l'étudiant est à risques d'échec ou s'il est en difficulté. La journée de la semaine et le lieu choisi pour la rencontre dépendent des disponibilités des deux parties. La rétroaction individuelle planifiée à mi-stage se déroule en milieu clinique dans un local dédié aux étudiants ou au cégep dans le bureau de l'enseignante. Les différentes motivations qui expliquent cette façon de rétroagir des participantes sont les suivantes : informer et situer l'étudiant dans la progression des éléments de compétences visés par le stage; lui rapporter des observations ; informer l'étudiant s'il est en danger d'échec ; échanger avec l'étudiant à propos des difficultés académiques ou personnelles et de solutions ; offrir l'aide nécessaire à l'étudiant pour qu'il s'améliore ; offrir une régulation à l'étudiant ; permettre à l'étudiant de s'autoévaluer ; apprécier la capacité de l'étudiant à s'autoévaluer et aider l'étudiant à progresser dans ses apprentissages. Une rétroaction individuelle planifiée à mi-stage est décrite comme suit par une enseignante: "II prend son rendez-vous, on a une rencontre, on discute. Donc, pendant la rencontre on va vérifier son champ de compétence. Je vais le mettre au courant des faits, de ce que j'ai observé, je vais reprendre aussi son travail de stage » (Part-03).

Les rétroactions individuelles planifiées peuvent se faire à la demande de l'étudiant, de façon régulière ou non. Pour ce faire, l'enseignante prend entente avec l'étudiant sur le lieu et le moment des rétroactions : " J'en avais comme une 


\section{Les pratiques évaluatives d'enseignants en soins infirmiers lors des stages : une étude descriptive qualitative}

[en parlant d'une étudiante] que je venais d'aviser et une qui était en supposé échec. On s'est entendu individuellement qu'on allait se parler après chaque quart de travail. Donc, après chaque quart, je lui disais qu'est-ce que j'avais aimé de sa journée, qu'est-ce qui avait été son amélioration, je ne focalisais pas sur les points négatifs, j'essaye d'y aller sur du positif, le négatif, ils l'ont déjà, ils le savent » (Part-05).

Une rétroaction individuelle planifiée de confrontation peut se pratiquer au besoin par les enseignantes. Avant de rencontrer l'étudiant en étant seule ou accompagnée d'une autre enseignante, l'enseignante consigne par écrit sur un contrat d'apprentissage les problèmes rencontrés par l'étudiant et les objectifs à poursuivre. Lors de la rencontre qui a lieu au cégep dans son bureau ou dans le bureau des coordonnatrices du département des soins infirmiers, l'étudiant est invité à se situer par rapport aux difficultés soulevées et à y apporter des solutions. Ensuite, pour signifier son accord et son engagement dans la résolution de la problématique soulevée, l'étudiant appose sa signature sur le contrat d'apprentissage. Cette pratique est employée lorsqu'un étudiant pose des difficultés, s'il a un problème récurrent ou s'il manque d'autocritique. Le but cité par les enseignantes est de laisser des traces des interventions et des observations faites pendant le stage. Une enseignante entretenue décrit cette pratique de la façon suivante : "Tu avises l'étudiante qu'elle doit venir te rencontrer et tu donnes un rendez-vous. Tu lui présentes le contrat, elle signe le contrat, elle est au courant, on lui explique pourquoi le contrat est fait, puis le contrat s'il n'est pas respecté cela peut-être un échec [...]. S'il y a vraiment un problème et qu'il faut absolument que je rencontre l'étudiant, lui faire signer un contrat ou peu importe vraiment des problèmes particuliers, à ce moment, je vais le faire venir au bureau » (Part-10).

Des rétroactions individuelles orales ponctuelles sont offertes à la demande de l'étudiant ou lorsque l'enseignante le juge nécessaire et que les conditions le permettent. Ces dernières sont réalisées la majorité du temps par l'enseignante. Elles sont réalisées à des moments variables, pendant ou après l'action de l'étudiant qui en faisait l'objet. Les lieux où elles sont faites sont variés, en présence ou pas du client, du personnel et des autres étudiants du groupe (chambre du client, poste des infirmières, corridor, bureau de l'enseignante). Les enseignantes interrogées motivent cette pratique des façons suivantes : pour soulever des éléments à corriger ou les éléments positifs ; lorsqu'un problème est répétitif ; lorsque le groupe est supervisé par plus d'une enseignante ; afin de solliciter des améliorations chez l'étudiant et s'assurer de la qualité des interventions et des soins. Une des participantes en fait la description de la façon suivante : "Cela peut être directement en sortant de la chambre si on est seul. Je pourrais lui dire : comment as-tu trouvé ta technique ou ton intervention $\mathrm{x}$ ? Cela peut être un enseignement aussi, ou peu importe. Et là, il va me dire soit c'était super ou oui je trouvais... Moi je vais dire : tu vois, moi, j'ai remarqué que ton asepsie ce n'est vraiment pas. Te rappelles-tu quels sont les 12 principes d'asepsie ? Là, il va en nommer deux, donc je vais lui dire de retourner dans tes volumes et tu vas retourner voir ce qu'est l'asepsie. Je ne le fais jamais en chicanant par exemple. Je pense que tout peut se dire, cela dépend de la façon que c'est dit. C'est ma façon d'intervenir à moi » (Part-04).

Les rétroactions individuelles ponctuelles peuvent aussi être réalisées par un autre étudiant du groupe (rétroaction par les pairs) et sont considérées comme une pratique évaluative puisqu'elles sont à la demande de l'enseignante. Lorsqu'un soin effectué par un étudiant est supervisé par un autre étudiant et que celui-ci lui fait des commentaires à propos du soin, cela constitue une rétroaction étudiant-étudiant : « L'étudiant, [...] peut enseigner à l'autre. Fais-le et moi je vais te reprendre. Donc, c'est lui qui va faire le «feed-back » si l'on veut, parce que quand tu es évaluateur, tu vois plus d'affaires des fois. Donc, il peut voir des choses que même lui il a oubliées ou de dire à l'autre : as-tu pensé le faire comme ça ? » (Part-04).

L'étudiant lui-même constitue une source de rétroaction lorsque l'enseignante, au lieu de lui adresser sa réaction, l'interroge à propos de sa perception face à sa réponse ou sa démarche (autorégulation et rétroaction interne). Parmi les enseignantes interrogées, l'une d'elles motive cette pratique de cette façon : "Pour moi, des fois ce que j'ai observé ce n'est pas tout à fait sa réalité à lui et j'aime bien cela avoir son point de vue. Et en plus, cela lui permet d'avoir une réflexion, donc cela amène une approche réflexive avec tout cela, avec l'étudiant, ce qui est super important » (Part-03).

Les rétroactions de groupe sont parfois planifiées ou elles surviennent au besoin et si les conditions le permettent. Les rétroactions de groupe ponctuelles correspondent aux commentaires de l'enseignante adressés à tout le groupe au sujet d'observations réalisées durant la journée de stage. Ce type de rétroaction est effectué de façon ponctuelle, souvent à la fin de la journée de stage, parfois le lendemain matin avant de débuter la journée de stage ou en après-midi. Pour ce faire, l'enseignante rassemble les étudiants du groupe dans un local dédié aux étudiants, dans la salle de repos des infirmières, dans une chambre non occupée par des clients, dans un corridor du milieu clinique ou dans un local dédié à d'autres vocations pour adresser sa rétroaction de façon verbale aux étudiants du groupe : "Je vais regrouper mes étudiants si cela ne fonctionne pas, s'il y a des problématiques générales dans le groupe, s'il y a des choses qui ne fonctionnent pas par rapport à la manière de fonctionner ou aux exigences du stage ou peu importe » (Part-09).

Le débriefing est rapporté comme une pratique de rétroaction de groupe non planifiée. II est réalisé de façon ponctuelle et variable selon les besoins du groupe et la faisabilité (temps et charge de travail). Un local dédié aux étudiants est utilisé à cet effet. Cette pratique amène la régulation des apprentissages 
et la régulation des méthodes d'enseignement : « Je vais les diriger, mais je vais les écouter aussi. Souvent, cela peut être des pleurs. Comment t'es-tu senti ? Comme ça, aujourd'hui, on a eu quelque chose de difficile. C'est les étudiants qui vont parler tout simplement. Je ne veux pas les laisser partir comme cela. [...] Au dernier stage, j'avais tellement des bons étudiants que je leur avais donné chacun un gros cas et finalement, je me suis rendu compte qu'un cas à deux, c'était suffisant parce que c'était vraiment très lourd, et le dossier et tout [...] il a fallu que je me traque ou que je m'ajuste ma façon » (Part-04).

Les rétroactions offertes lors de rencontre de groupe sont planifiées ou non, et elles se déroulent dans un local fermé dans le milieu clinique. Des échanges ont lieu entre l'enseignante et les étudiants à propos d'événements passés dans le but d'amasser des informations qui sont nécessaires à l'évaluation, d'améliorer la compréhension de l'étudiant, de situer l'étudiant dans ses apprentissages et de permettre à l'étudiant de s'améliorer. Une participante explique cette pratique comme suit : " Je vais essayer peut-être à la fin des stages, une fois peut-être à mi-stage et ce n'est pas long ça peut-être 30 minutes [...]. On va se rassembler et on va revenir sur les cas importants. Comment on a fonctionné, comment on pourrait faire et comment je verrais la suite des choses. Ou [ ] comment j'ai trouvé leur façon de fonctionner, qu'est-ce que je voudrais qu'ils fassent. ce que j'ai remarqué par rapport au groupe en général » (Part-04).

\section{Outils d'évaluation}

Les outils d'évaluation utilisés afin d'aider l'enseignante à recueillir et obtenir des informations sur le développement des compétences de l'étudiant sont les suivants : les exercices et les mises en situation lors de rencontres au début du stage ; la liste de vérification ; la rédaction de notes (cahier, carnet et feuille de route) ; le journal de bord de l'enseignant ; la liste des enseignements prodigués et le contrat d'apprentissage.

Les pratiques déclarées confirment que les enseignantes consignent leurs observations. Cependant, même si elles les consignent, elles le font de façons variées et peu d'entre elles le font de façon continue. Cette pratique varie beaucoup en termes de fréquence et de rigueur. Parfois, les notes sont prises immédiatement après l'observation, mais la plupart du temps, elles sont prises de façon différée.

Les outils d'évaluation utilisés afin de documenter la performance de l'étudiant sont : la grille d'évaluation formative et sommative et la grille d'évaluation hebdomadaire.

\section{Facteurs d'influence des pratiques évaluatives des enseignants lors de l'évaluation en cours d'apprentissage d'étudiants en contexte de stage}

Les entrevues réalisées auprès des enseignantes ont aussi permis d'identifier certains facteurs qui, selon ces dernières, influencent le choix des pratiques évaluatives, et la façon de les mettre en œuvre. Ces facteurs ont été répertoriés en trois groupes soit : les facteurs facilitants, les barrières et les croyances. Les facteurs facilitants et les barrières sont présentés et regroupés selon les catégories suivantes : organisation du stage, milieu, méthodes et outils employés par l'enseignant, caractéristiques de l'étudiant, caractéristiques de l'enseignant et caractéristiques de client-famille (tableau 2). Une enseignante discute du fait d'avoir un groupe de cinq étudiants en comparaison d'un groupe de six étudiants dans l'extrait suivant : “C'est parce que je vois mieux, je vois plus, j'ai plus de temps à leur accorder et j'ai l'impression d'avoir plus le temps de leur parler. Car à six étudiants, on éteint des feux » (Part-01). Par ailleurs, certaines croyances, qui ont été évoquées pendant les entretiens, sont susceptibles d'influencer les pratiques évaluatives des enseignants dans ce contexte précis. II ressort des propos des participantes les croyances suivantes : l'étudiant doit être au cœur de ses apprentissages ; la progression de l'étudiant dans ses apprentissages est importante et doit être visée dans les pratiques évaluatives ; la relation de confiance entre l'étudiant et l'enseignant est importante ; la formation en pédagogie aide l'enseignant dans ses pratiques évaluatives ; le devis ministériel n'a pas été établi en fonction de la réalité du marché du travail actuel ; l'objet de l'évaluation en stage comprend plus que l'aspect technique et il comprend entre autres le jugement clinique, l'évaluation des attitudes ; l'évaluation est intuitive. Voici un extrait d'entrevue reflétant la croyance à l'effet que la formation en pédagogie aide l'enseignant dans ses pratiques évaluatives : «Donc je pense que plus nous sommes outillés [en parlant des enseignantes], plus nous avons de la formation en tant que pédagogue, plus nous comprenons notre rôle professionnel, le champ professionnel de la pratique dans laquelle nous devons sanctionner [...], plus que tu comprends, décortiques tes compétences professionnelles je pense que ça a un impact [en parlant des pratiques évaluatives]. Et cela se fait par le biais de la formation en pédagogie » (Part-03).

\section{DISCUSSION}

La discussion proposée aborde les différentes pratiques évaluatives mises en œuvre en y intégrant les facteurs d'influence et les croyances au fur et à mesure. Les outils mentionnés par les participants sont également discutés.

\section{Pratiques de collecte d'information}

Plusieurs facteurs limitent le temps accordé à la collecte d'information par l'observation (directe et indirecte) et les questions posées à l'étudiant ou au groupe d'étudiants. En effet, les résultats à cet effet convergent vers ceux proposés par l'étude de Desrosiers (4) à savoir que le contexte de soins, les milieux de stages et le trop grand nombre d'étudiants à encadrer sont des obstacles à l'encadrement 


\begin{tabular}{|c|c|c|}
\hline & Facteurs facilitants & Barrières \\
\hline 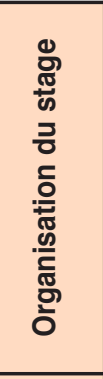 & $\begin{array}{l}\text { - Nombre d'étudiants supervisés inférieur } \\
\text { ou égal à cinq ; } \\
\text { - Stages de collaboration avec les } \\
\text { infirmières auxiliaires; } \\
\text { - Supervision effectuée par deux } \\
\text { enseignants; } \\
\text { - Supervision de plusieurs jours consécutifs ; } \\
\text { - Durée du stage de cinq semaines ; } \\
\text { - Disponibilité de l'enseignant pour partager } \\
\text { l'information relative à l'évaluation. } \\
\end{array}$ & $\begin{array}{l}\text { - Répartition de la théorie et des laboratoires dans une session (pas } \\
\text { préalables aux stages); } \\
\text { - Charge de travail très élevée de l'enseignant ; } \\
\text { - Peu de temps disponible relié à la lourdeur des soins du milieu } \\
\text { clinique; } \\
\text { - Nombre d'étudiants par groupe [6]; } \\
\text { - Mode d'attribution des étudiants aux infirmières et au(x) client(s) } \\
\text { lors d'un stage; } \\
\text { - Encadrement de plus d'une enseignante pour un stage. }\end{array}$ \\
\hline 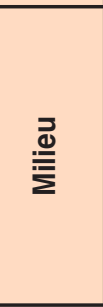 & $\begin{array}{l}\text { - Salle adaptée et dédiée aux étudiants } \\
\text { dans le milieu clinique ; } \\
\text { - Milieu clinique où la lourdeur de travail } \\
\text { est moindre. }\end{array}$ & $\begin{array}{ll}\text { - } & \text { Lieux physiques manquant d'espace et d'intimité pour rencontrer } \\
& \text { les étudiants ; } \\
\text { - } & \text { Chambre du client avec plusieurs lits ; } \\
\text { - } & \text { Rapidité du milieu; } \\
\text { - } & \text { Nécessité d'intervenir rapidement dans certaines situations ; } \\
\text { - } & \text { Absence de salle dédiée aux étudiants et disponible dans le } \\
& \text { milieu clinique ; } \\
\text { - } & \text { Milieu clinique où la charge de travail est élevée (lourdeur de la tâche). }\end{array}$ \\
\hline 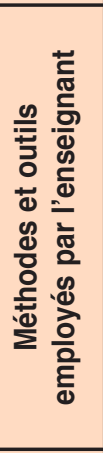 & $\begin{array}{l}\text { - En début de stage, limiter le nombre de } \\
\text { clients à l'étudiant ; } \\
\text { - Nombre moindre de clients attribués aux } \\
\text { - étudiants ; } \\
\text { - Avis d'une autre enseignante; } \\
\text { - Consignation des observations en notes; } \\
\text { - Démarche de soins contenue dans les } \\
\text { - travaux de stage; } \\
\text { - Situation d'évaluation constituant une } \\
\text { situation complexe (client nécessitant } \\
\text { beaucoup de soins et surveillances). }\end{array}$ & 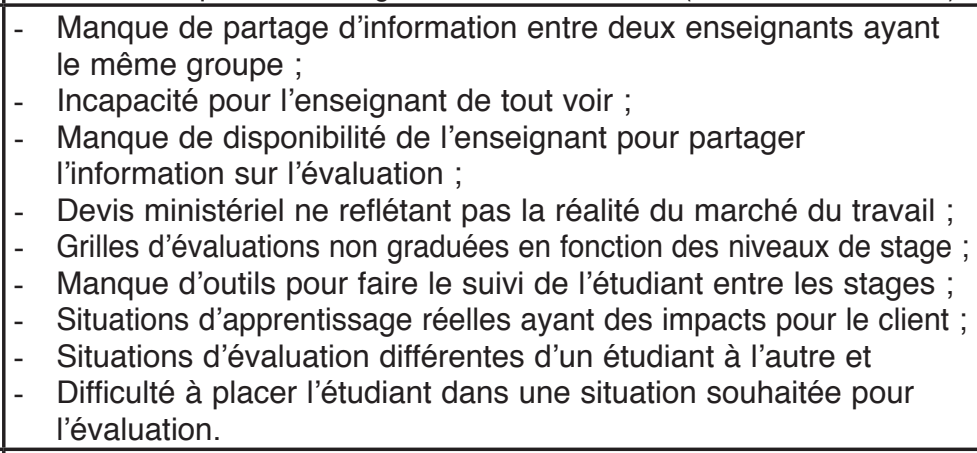 \\
\hline 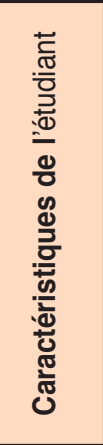 & $\begin{array}{l}\text { - Enseigner à un étudiant motivé et } \\
\text { engagé. }\end{array}$ & $\begin{array}{l}\text { - Groupe d'étudiants non homogène (ratio étudiants forts/faibles) ; } \\
\text { - Groupe d'étudiant comprenant un étudiant en difficulté ; } \\
\text { - Enseigner à un étudiant qui ne progresse pas au même rythme } \\
\text { que les autres étudiants du groupe ; } \\
\text { - Enseigner à plus d'un étudiant problématique dans un groupe de } \\
\text { stage ; } \\
\text { - Enseigner à un étudiant anxieux ; } \\
\text { - Manque de responsabilité de l'étudiant (ne prends pas rendez- } \\
\text { vous); } \\
\text { - Enseigner à un groupe d'étudiant de première année ; } \\
-\quad \text { Enseigner à un étudiant émotif ; } \\
-\quad \text { Manque de disponibilité de l'étudiant. }\end{array}$ \\
\hline 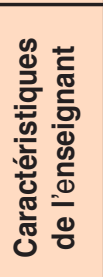 & $\begin{array}{l}\text { - Compréhension de l'Approche par } \\
\text { Compétence (APC) ; } \\
\text { - Forme physique de l'enseignant. }\end{array}$ & $\begin{array}{l}\text { - Crainte de l'enseignant d'entraver la confiance en soi de l'étudiant. } \\
\text { - Fatigue engendrée par l'encadrement des étudiants en stage ; } \\
\text { - Corrections et annotations faites dans les travaux de recherches } \\
\text { ne sont pas faits selon les mêmes barèmes d'un enseignant à } \\
\text { l'autre; } \\
\text { - Hétérogénéité dans la formation en pédagogie reçue par les } \\
\text { enseignants du département. }\end{array}$ \\
\hline 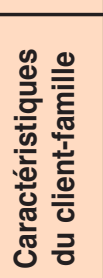 & $\begin{array}{l}\text { - Bonne collaboration du client et de la } \\
\text { famille lors du partage d'information. }\end{array}$ & $\begin{array}{l}\text { - État du client qui peut l'empêcher de collaborer dans le partage } \\
\text { d'information. }\end{array}$ \\
\hline
\end{tabular}

Tableau 2:

Facteurs d'influence des pratiques évaluatives lors de l'évaluation en cours d'apprentissage d'étudiants en stages de soins infirmiers 
fait par les enseignants en stage. Dans la présente étude, les enseignantes interrogées soulèvent que le milieu et l'organisation du stage sont des barrières à l'observation. Les travaux de Théorêt et Saint-Pierre étudiant les stages en formation universitaire (supervision indirecte), soulèvent aussi l'importance du climat d'apprentissage et la relation de confiance entre l'étudiant et son moniteur de stage (20). Ces résultats vont dans le même sens que les propos de St-Pierre qui soulève que le stress de l'étudiant est un facteur à considérer dans l'encadrement en stage lors de formation infirmière universitaire (21).

Par ailleurs, il a été mentionné par les participants que les étudiants ayant des cas complexes, les étudiants en difficulté et les étudiants faisant des actes à risques d'erreur ou ayant un impact important sur le client sont observés plus souvent. Ces situations nécessitent un encadrement plus important de l'enseignante. Conséquemment, lorsqu'un groupe de stage comprend un étudiant en difficulté, celui-ci nécessite plus de temps d'observation et d'encadrement, ce qui a comme impact d'en laisser moins pour les autres étudiants. II a été soulevé aussi que ce problème est accentué lorsque le groupe comprend plus d'un étudiant problématique. II en résulte que les informations amassées au sujet des autres étudiants du groupe sont moindres et peuvent mettre en doute l'évaluation.

Plusieurs enseignantes interrogées mentionnent que le peu de temps disponible pour la collecte d'information est une conséquence de la surcharge de travail imposée par le milieu, les soins aux clients et le nombre trop grand d'étudiants. De surcroît, lorsque des étudiants sont jumelés à des infirmières d'un milieu très actif (par exemple aux urgences), cela diminue la fréquence et la durée accordée à l'observation directe de l'étudiant. Au contraire, lorsque le milieu clinique entraîne une charge de travail moindre, les pratiques évaluatives (collecte d'informations et rétroaction) sont favorisées. Par exemple, un milieu tel une unité de médecine où la charge de travail est moindre facilite la mise en œuvre des pratiques évaluatives des enseignantes, et ce, contrairement à un milieu comme une unité de chirurgie où la charge de travail est plus grande et qui constitue plutôt une barrière.

\section{Pratiques de rétroaction}

Par ailleurs, les pratiques de rétroaction rapportées par les enseignantes se rapprochent des résultats apportés par l'étude de Desrosiers (4). En effet, cette étude identifie les stratégies suivantes favorisant la rétroaction et la pratique réflexive : « la rétroaction immédiate ou planifiée, la résolution de problème, le modelage, les rencontres d'évaluation formatives et sommatives avec questionnements » (4, p.79). Les descriptions des pratiques de rétroaction recensées permettent d'affirmer que les enseignantes procèdent de façon variée en regard de la nature du contenu, soit la présence d'aspects positifs, d'aspects négatifs et d'indices permettant à l'étudiant de se reprendre. Certaines enseignantes fournissent des explications et des indices pour que l'étudiant se corrige, mais la majorité ne donne qu'une rétroaction simple.

Plusieurs enseignantes ont soulevé que le temps et la surcharge de travail sont des obstacles à la rétroaction, plus particulièrement un obstacle à offrir une rétroaction rapidement après l'action. Les situations dans lesquelles l'étudiant est placé ne permettent pas, selon la plupart des enseignantes, de donner une rétroaction pendant l'action. Elles motivent cette limitation par le désir de ne pas entraver la relation entre l'étudiant et le client. Par contre, dans certaines situations et pour certaines, il arrive que l'enseignante intervienne immédiatement. Parfois, elles doivent reprendre la situation en main pour maintenir la qualité des soins au client ou éviter un accident. Les rétroactions pendant l'action sont moins fréquentes et sont plutôt positives. Alors que les rétroactions faites de façon générale par les participantes sont pour la plupart orientées sur les aspects négatifs. Certaines des personnes entretenues motivent la nature des rétroactions employées (simple et négative) par la surcharge de travail.

\section{Outils d'évaluation}

Les écrits recommandent de noter au fur et à mesure ce qui est observé afin d'évaluer un étudiant en stage (1). Mais encore, pour le faire de façon structurée, ils suggèrent d'utiliser un cahier de notes dans lequel l'enseignant prend des notes pour chaque étudiant (1). Subséquemment, il apparaît un décalage entre ce qui est recommandé et les pratiques effectives des enseignantes interrogées. II semble que les enseignantes se servent, certes, de l'observation directe, mais que la plupart le font de façon intuitive et subjective, puisque la plupart n'utilisent pas d'outils d'observation formels et que les notes d'observation ne sont pas prises au fur et à mesure.

La grille d'évaluation a souvent été mentionnée par les participantes comme étant une barrière à l'évaluation. Elle est composée principalement de critères et d'une échelle d'appréciation uniforme numérique (graduée de un à cinq). Les enseignantes ont mentionné que cette grille est " déficiente ", qu'elle n'est pas graduée en fonction des niveaux de stage, et qu'elle ne comporte pas les savoir-être. Alors que l'évaluation des tâches complexes nécessite l'utilisation de plusieurs types de grilles d'évaluation, permettant d'exercer son jugement, que ce soit lors d'évaluations formative ou certificative (22), un seul type de grille n'est utilisé par les enseignantes participantes pour l'évaluation en cours de stage et l'évaluation à la fin du stage (certificative).

\section{Forces et limites de l'étude}

Cette étude comprend des forces en regard de certains choix méthodologiques et de ses résultats. En effet, elle permet de décrire en profondeur les pratiques évaluatives d'enseignants lors de l'évaluation en cours d'apprentissage d'étudiants en stage de soins infirmiers, chose qui, selon ce qui a été trouvé 


\section{Les pratiques évaluatives d'enseignants en soins infirmiers lors des stages : une étude descriptive qualitative}

dans les écrits, existait peu jusqu'à aujourd'hui. La saturation des données, moyen pour assurer la fiabilité des données, ne s'évalue pas en fonction du nombre de participants, mais est plutôt une question du jugement (23-25). Ainsi, les 10 entrevues initiales et les 10 entrevues de validation réalisées semblent avoir permis d'atteindre une saturation empirique. Les deux dernières entrevues initiales effectuées n'ont pas ajouté « d'informations suffisamment nouvelles ou différentes » (25, p.157) et c'est pourquoi le niveau de saturation empirique semble avoir été atteint. Puisque l'interviewer a réalisé la totalité des entrevues, des retranscriptions et l'encodage, cela lui a permis d'ajuster les questions du guide pour explorer davantage certains aspects (exploration en profondeur) et de s'imprégner des données. Par conséquent, les données sont d'autant plus riches et l'on peut supposer que le processus d'analyse a été optimisé par ce processus itératif. En plus, la validation des résultats a permis, entre autres, de renforcer la rigueur inhérente à cette étude. De plus, cette deuxième entrevue a permis aux participantes d'ajouter des propos auxquels elles n'avaient pas pensé lors de l'entrevue initiale. Finalement, les entrevues réalisées auprès des participantes ont su amorcer une réflexion à propos de leurs pratiques évaluatives faites lors des stages.

Les limites possibles et inhérentes à cette étude comprennent entre autres celles reliées aux choix méthodologiques. La première se rapporte à la méthode de collecte de données qui ne comprend qu'une source de données provenant d'entrevues alors qu'elle aurait pu exploiter d'autres méthodes telles que l'observation et l'analyse d'outils d'évaluation. La deuxième limite concerne le fait que les données obtenues sont des pratiques autodéclarées. La véracité des pratiques déclarées pourrait être questionnée à savoir : est-ce qu'elles correspondent aux pratiques réelles faites sur le terrain ? Des observations qui auraient eu lieu lors d'entretiens d'évaluation entre les enseignantes et les étudiants et lors d'enseignements cliniques auraient sans doute amélioré la crédibilité de l'étude. La conduite d'entrevues avec les étudiants évalués lors des stages auraient également renforcé ce critère. Un autre choix méthodologique pouvant limiter l'étude est le milieu choisi pour le recrutement des participants qui comprend un seul établissement.

\section{CONCLUSION}

Cette étude descriptive qualitative a eu comme visée de décrire les pratiques évaluatives d'enseignants lors de l'évaluation en cours d'apprentissage auprès des étudiants en stage et leur contexte de réalisation ainsi qu'à identifier les facteurs d'influence de ces pratiques. Par la description de leur contexte de réalisation ainsi que des facteurs facilitants, des barrières et des croyances, une meilleure compréhension de ces pratiques a été obtenue. II en ressort que la mise en œuvre des pratiques évaluatives par les enseignantes dans le contexte étudié sont multiples et variées. Le contexte de réalisation et le milieu constituent les barrières majeures pour les pratiques de collecte d'information et de rétroaction effectives. Toutefois, il faut considérer que "l'expérience de travail, l'adaptation aux caractéristiques de l'étudiant, l'action enseignante et les pratiques enseignantes évoluent au même titre que les pratiques évaluatives » $(26$, p.235-6). L'étude offre tout de même un premier portrait de ce qui est fait actuellement dans un cégep de la région du SLSJ et peut alors amorcer d'autres projets de recherches futures à ce sujet. Elle soulève la problématique reliée à la formation en pédagogie des enseignants au collégial du Québec. Les données descriptives recueillies corroborent les écrits à l'effet qu'aucune formation en pédagogie n'est exigée des enseignants en formation collégiale et que celle reçue par les enseignants québécois est très variée. Ainsi, la question de la formation en pédagogie chez les enseignants en formation collégiale demeure.

\section{FINANCEMENTS ET DÉCLARATION DE CONFLITS D'INTÉRÊTS}

Les auteurs déclarent n'avoir aucun lien d'intérêt.

\section{Références}

1. Ménard L, Gosselin R. Évaluer sur le terrain et dans l'action : de nombreux défis. Dans: Leroux JL, dir. Évaluer les compétences au collégial et à l'université : un guide pratique. Montréal: Association québécoise de pédagogie collégiale (AQPC); 2015. p. $577-627$.

2. Phaneuf M. L'apprentissage/enseignement en milieu clinique [En ligne]. Prendre soin; 2012. [cité le 25 octobre 2016]. Disponible: http ://www.prendresoin.org/?p=2164

3. Ministère de l'Éducation du Loisir et du Sport. Programme d'étude technique: soins infirmiers. Québec: Gouvernement du Québec; 2007.

4. Desrosiers F. Étude des pratiques de supervision de stage dans un programme par compétences en soins infirmiers au collégial. [Essai de maîtrise en enseignement au collégial]. Sherbrooke: Université de Sherbrooke; 2009.

5. Villeneuve L. L'encadrement du stage supervisé. Montréal: Éditions Saint-Martin; 1994.

6. Fortin MF, Gagnon J. Fondements et étapes du processus de recherche: méthodes quantitatives et qualitatives. 3e éd. Montréal: Chenelière éducation; 2016.

7. Sandeloski M. Whatever happened to qualitative description? Res Nurs Health. 2000 Aug:23(4):334-40.

8. Holloway I, Wheller S. Qualitative research in nursing and healthcare. 3e éd. Oxford: Wiley-Blackwell; 2010. 
9. Laperrière A. Les critères de scientificité des méthodes qualitatives. Dans: Groupe de recherche interdisciplinaire sur les méthodes qualitatives, directeurs. La recherche qualitative : enjeux épistémologiques et méthodologiques. Montréal: QC: Gaëtan Morin éditeur; 1997. p. 365-89.

10. Guest G, Namey EE, Mitchell ML. Collecting qualitative data: a field manual for applied research. Thousand Oaks: SAGE Publications Inc.; 2013.

11. Miles MB, Huberman AM, Saldaña J. Qualitative data analysis: a methods sourcebook. 3e éd. Thousand Oaks: SAGE Publications Inc.; 2014.

12. Streubert HJ, Carpenter DR. Qualitative research in nursing: advancing the humanistic imperative. 5e éd. Philadelphia: Lippincott Williams \& Wilkins; 2011.

13. Roland L. L'évaluation des apprentissages en classe : théorie et pratique. Laval: Beauchemin éditeurs; 1999.

14. De Ketele JM. Méthodologie de I'observation. Bruxelles: De Boeck; 1988.

15. De Ketele JM. Observer pour éduquer. 4e éd. Berne: Édition Peter Lang SA; 1987.

16. De Ketele JM, Roegiers X. Méthodologie du recueil d'informations: fondements des méthodes d'observation, de questionnaire, d'interview et d'étude de documents. 5e éd. Bruxelles: De Boeck; 2015.

17. Scallon G. L'évaluation des apprentissages dans une approche par compétences. Québec: Éditions du Renouveau Pédagogique; 2004.

18. Allal L, Mottiez Lopez L. Régulation des apprentissages en situation scolaire et en formation. Bruxelles: De Boeck; 2007.
19. Leroux JL. Évaluer pour faire apprendre. Dans: Ménard L, St-Pierre $\mathrm{L}$, directeurs. Se former à la pédagogie de l'enseignement supérieur. Montréal, QC: Association québécoise de pédagogie collégiale (AQPC); 2014. p. 333-53.

20. Théorêt L, Saint-Pierre C. L'importance du climat d'apprentissage en milieu de stage. Dans: St-Pierre L, directeur. Se former pour mieux superviser en sciences infirmières. Montréal, QC: Beauchemin; 2007.

21. St-Pierre L. L'importance du stress vécu par les stagaires en formation pratique. Dans: St-Pierre L, directeur. Se former pour mieux superviser en sciences infirmières. Montréal, QC: Beauchemin; 2007. p. 56-65.

22. Leroux JL. Analyse des pratiques évaluatives d'enseignantes et d'enseignants dans une approche par compétences au collégial. [Thèse de doctorat inédite]. Sherbrooke: Université de Sherbrooke; 2009.

23. O’Reilly M, Parker N. 'Unsatisfactory Saturation': a critical exploration of the notion of saturated sample sizes in qualitative research. Qualitative Research. 2012 May;13(2):190-7.

24. Morse J. Critical Analysis of Strategies for Determining Rigor in Qualitative Inquiry. Qual Health Res. 2015 Sep;25(9):121222.

25. Sandelowski M. Sample size in qualitative research. Res Nurs Health. 1995 Apr;18(2):179-83.

26. Clanet J. Rapport entre les pratiques enseignantes et la progression des élèves dans leurs apprentissages : nouvelles appréhensions. Dans: Altet M, Bru M, Blanchard-Laville C, directeurs. Observer les pratiques enseignantes. Paris: L'Harmattan; 2012. p. 223-38. 